\title{
The Energization of Cold Ions around a Plasmoid in the Magnetotail
}

\author{
Ken Tsubouchi and Toshio Terasawa \\ Department of Earth and Planetary Physics, The University of Tokyo, \\ Hongo 7-3-1, Bunkyo-ku, Tokyo 113, Japan
}

(Received July 18, 1994; Revised October 20, 1994; Accepted November 21, 1994)

\begin{abstract}
We study numerically the interaction of cold ionospheric ions in the tail lobe with a plasmoid propagating steadily in the tailward direction. We have found that significant energization of cold ions occurs at the encounter with a plasmoid, where three types of the interaction processes are taking place: First, ions show gradient and curvature drift motions as the plasmoid approaches. These ions are accelerated up to $\sim 1 \mathrm{keV}$ by the cross-tail electric field accompanying with the plasmoid motion (type-I energization). Second, some of the ions get large pitch angles after the type-I acceleration, and then mirror reflected by the plasmoid. These ions are accelerated up to $10 \mathrm{keV}$ after the reflection (type-II energization). Third, the motions of the ions near the plasmoid become highly non-adiabatic, because their gyroradii become comparable to the field-line curvature radius after the type-I energization. Consequently the energy distribution of these ions is broadened in the trailing side of the plasmoid (type-III energization). These theoretical results are successfully compared with the recent GEOTAIL observation showing the dynamic interaction of cold ions with a plasmoid and their subsequent energization.
\end{abstract}

\section{Introduction}

Since the discovery of the magnetotail, extensive studies on its structure and dynamics have been done. A remarkable improvement on the instruments onboard the spacecraft makes it possible to describe a detailed configuration and dynamics of the distant magnetotail. The ISEE 3 geotail observations have made great advances in the comprehension of the dynamical process in the distant magnetotail (e.g. Hones et al., 1984), leading to the establishment of the 'plasmoid' model (Hones, 1977). The main signatures of plasmoids are, (1) fast, anti-sunward flow ( $\sim 700$ $\mathrm{km} / \mathrm{s}$ ) in the central plasma sheet (Baker et al., 1987), (2) north-then-south tilting of the plasma sheet magnetic field (Baker et al., 1987), (3) energetic charged particles with isotropic pitch angle distributions in the plasma rest frame (Scholer et al., 1984), (4) transient enhancement of lobe magnetic field ('traveling compression regions', or TCRs (Slavin et al., 1984). For the reviews on the detailed plasmoid analysis, see Slavin et al. (1993) and the references therein.

Scholer et al. (1984) presented a detailed study of the temporal behavior of energetic protons and electrons observed in association with plasmoid passages. They discussed that these energetic particles ( $\geq$ a few tens of $\mathrm{keV}$ ) are produced by the same magnetic reconnection process as that responsible for the plasmoid formation. Sato et al. (1982) and Scholer and Jamitzky (1987) followed numerically the trajectories of particles in the electric and magnetic field obtained from a two-dimensional MHD simulation of reconnection. These studies show the complexity and importance of the particle motion near the neutral sheet, where the adiabaticity of the particle motion is easily violated. Calculations of test particle trajectories in stationary neutral sheet were also studied by several authors (Speiser, 1965; Sonnerup, 1971; Wagner et al., 1979; Gray and Lee, 1982). Wagner et al. have classified the particle orbits into adiabatic, transitional, and non-adiabatic case, according to the field geometry. In their results, particles in adiabatic motion experience no cross-tail drift (i.e. no net encrgization) and the final pitch angle is close to the 
initial value, while non-adiabatic orbits undergo a net cross-tail displacement as they pass through the plasma sheet (gain energy), and initial and final pitch angles lose their relationship. Gray and Lee (1982) have shown that for a large value of $\rho / R_{c}$ the random change in the magnetic moment takes place as a particle passes the midplane of the plasma sheet. ( $R_{c}$ is the characteristic length over which the field varies, here the radius of the curvature, and $\rho$ the gyroradius of the particle.) This change brings about stochastic motion of particles. After these processes particles very well become isotropic.

We now turn our attention to the lower energy ions. Existence of cold ions of ionospheric origin is proved to be a semi-persistent feature in the tail lobe (Shelley et al., 1976; Frank et al., 1977; Hardy et al., 1977; Sharp et al., 1981; Mukai et al., 1994). We expect that the motions of these cold ions are significantly perturbed during the magnetically disturbed period. The GEOTAIL plasma observation has given the observational confirmation of this expectation

(a)

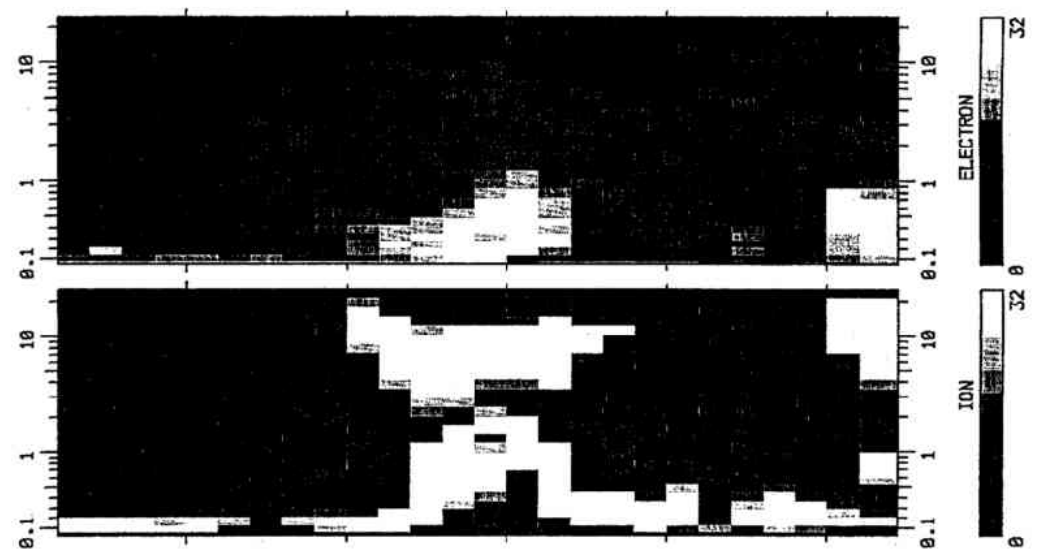

(b)

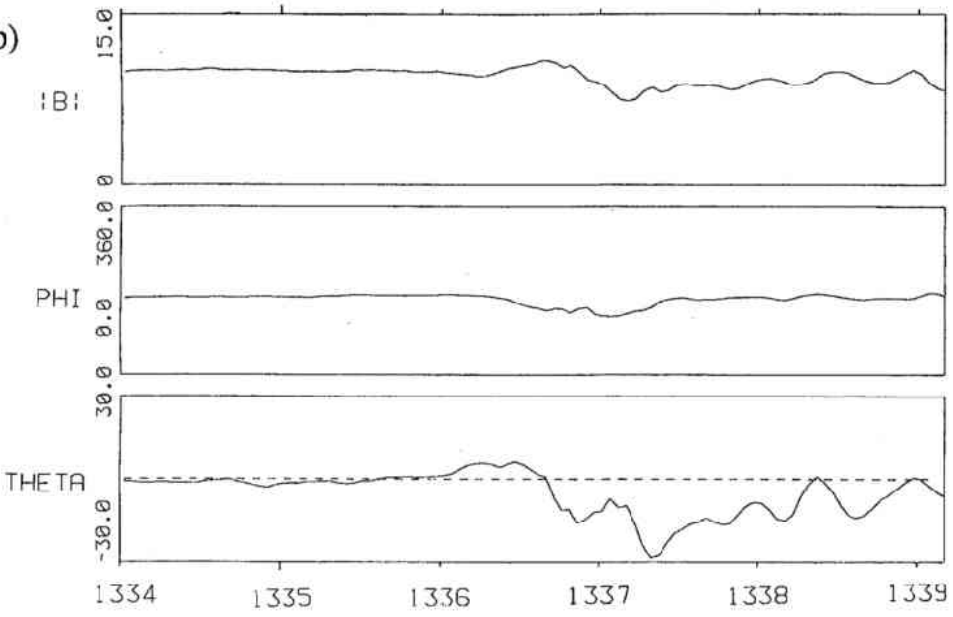

Fig. 1. (a) Ion energy-time spectrogram with GEOTAIL LEP for the period 1334-1339 UT on September 17, 1993 at $(X, Y, Z) \sim(-73,+7,0) \mathrm{R}_{\mathrm{E}}$ in GSM coordinates, for electron (top) and ion (bottom). The values indicated in the ordinate represent the energy of $\mathrm{keV}$, and the gray code shows counts/sample (see the right hand scale). (b) GEOTAIL magnetic field observations simultaneous with the particle observations in Fig. 1(a) are displayed: From the top to the bottom, total magnitude of $|B|$, the azimuthal angle $\phi$, and the elevation angle $\theta$ ( $\phi$ and $\theta$ are in the GSM coordinate). 
(Mukai et al., 1994; Hirahara et al., 1994). Mukai et al. (1994) reported that the cold ions were significantly heated shortly before and after the passage of a plasmoid. Another example of the plasmoid-cold ion interaction is given in Fig. 1(a), where energy-time spectrograms of electrons (upper panel) and ions (lower panel) are shown. Figure 1(b) shows the total magnitude (upper), the azimuthal angle (middle), and the elevation angle (lower) of the magnetic field during the interval corresponding to Fig. 1(a). When the spacecraft was in the lobe region ( $1336 \mathrm{UT})$, there were the ion streams at energies of $\sim 100 \mathrm{eV}$ with a narrow energy width, i.e. cold temperature. The flow direction was approximately tailward, parallel to the local magnetic field. During the interval of 1336 1339 UT, the field elevation angle $\theta$ showed a north $(\theta>0)$-then-south $(\theta<0)$ excursion. The total field magnitude was maximized at $\sim 1336: 40$ UT near the inflection point $(\theta \sim 0)$ (Fig. 1(b)); these signatures are identified as the encounter of the plasmoid or TCR. Ions with the energy range above $3 \mathrm{keV}$ during 1336:00 1337:48 UT in Fig. 1(a) constitute this plasmoid. The cold ions in Fig. 1(a) showed significant variations through the plasmoid passage: (1) the enhancement of the ion energies up to $\sim 2 \mathrm{keV}$ while passing the plasmoid proper (1336 1337 UT), and (2) the widely spread energy distribution (heating) after the plasmoid passage (1337:24 1338:12 UT).

The purpose of this study is to examine the behavior of cold ions in the region surrounding a plasmoid in order to explain the observed feature stated above. Our model is schematically drawn in Fig. 2: At first a spacecraft is in the lobe field and detects cold ions streaming tailward with a bulk velocity of $\sim 100 \mathrm{~km} / \mathrm{s}$. Then the spacecraft meets the closed field region of a plasmoid moving tailward with the velocity of $\sim 700 \mathrm{~km} / \mathrm{s}$, and finally (after $\sim 60 \mathrm{~s}$ ) exits from the plasmoid back to the lobe region. The plan of the present paper is as follows. In Section 2, we introduce the magnetic field model derived by Birn (1992), as well as the orbit calculation scheme. The results of the test particle simulation are given in Section 3 . The results are summarized in Section 4.

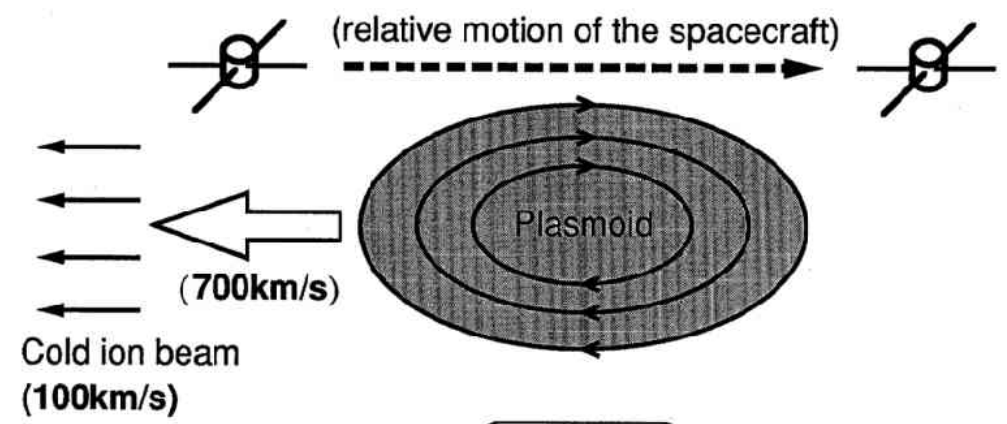

$\longleftarrow$ tailward

Fig. 2. A schematic diagram, which depicts the situation of the GEOTAIL traveling during the period 1336-1339 UT on September 17, 1993, in order to be consistent with the observations.

\section{Calculation Scheme}

\subsection{Model magnetic field}

In order to follow the trajectories of test particles at a plasmoid encounter, we need to set a background field configuration. In the present study, we choose a two-dimensional plasmoid model numerically obtained by Birn (1992). This Birn's model is made in the 'de Hoffmann-Teller (dHT) frame', in which the electric field vanishes $(\boldsymbol{E}=0)$ and the plasmoid has a quasi-steady, time-independent structure with a field aligned plasma flow (Fig. 3). This model is based on the 

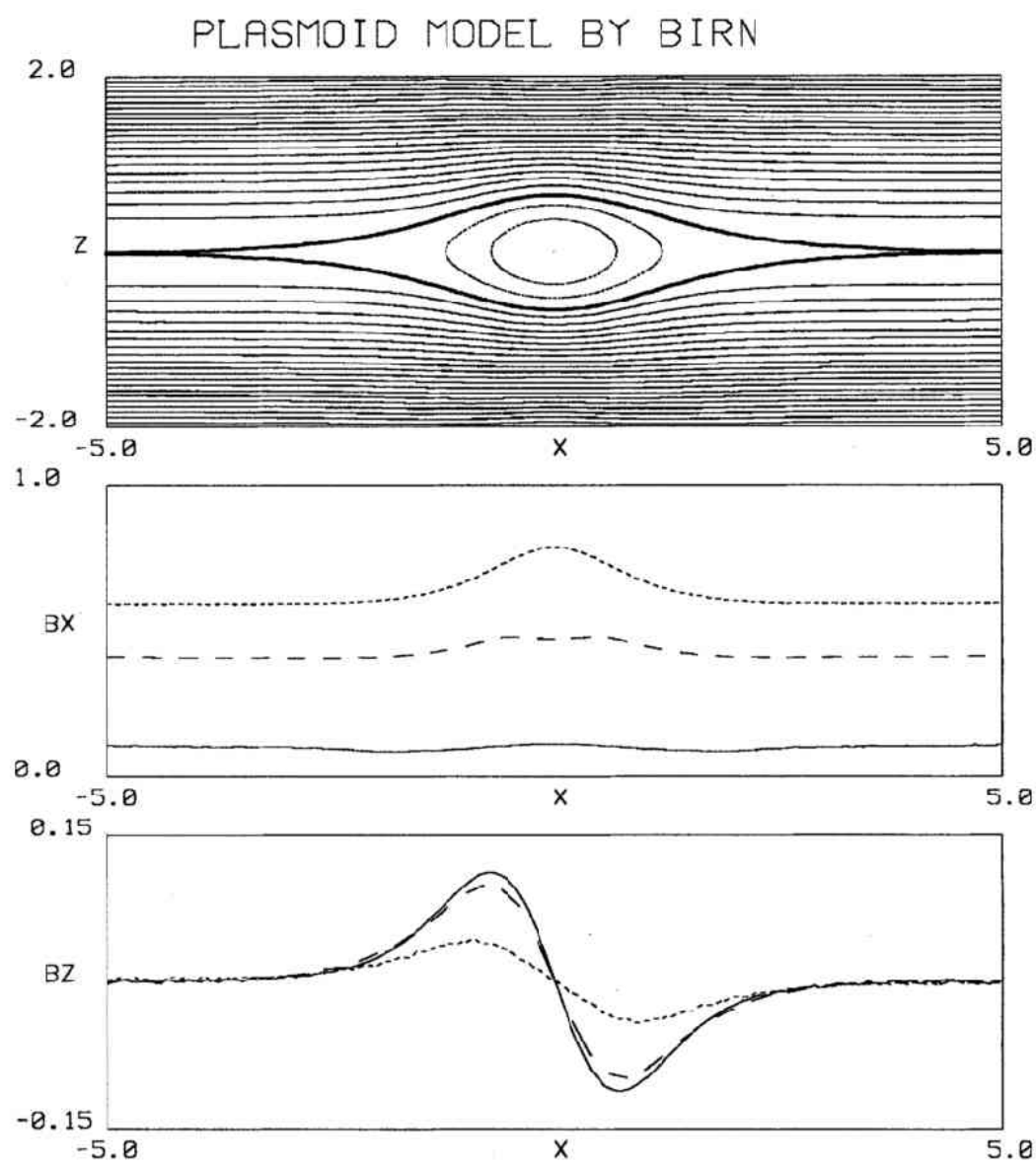

Fig. 3. (Top): Magnetic field line configuration derived from Birn's model (Birn, 1992), embedded in the present computational box. The heavy line represents the plasmoid boundary. The length scale $L_{x}$ and $L_{z}$ can be adjusted independently. Variation of (middle): $B_{x}$ and (bottom): $B_{z}$ with $x$ at $z=0.2$ (a solid line), 0.71 (a dashed line), and 1.2 (a broken line).

assumption that plasma and field gradients along the $x$ direction parallel to the tail current sheet are much smaller than those in the $z$ direction perpendicular to the current sheet. Here the $x$ axis lies along the earth-sun line (positive towards the sun), and the $z$ axis along the south-to-north line.

The top panel of Fig. 3 shows the magnetic field lines for a plasmoid in the simulation box. The heavy curves represent the boundary of the plasmoid, i.e. the separatrices between closed loop-like field lines and open field lines. The middle and the bottom panels of Fig. 3, respectively, show the variation of $B_{x}$ and $B_{z}$ with $x$, which is to be observed as time profiles on a spacecraft at $z=0.2,0.71$, and 1.2. The enhancement of $B_{x}$ in the center of the plasmoid accompanying the first northward then southward magnetic field $\left(B_{z}\right)$ excursion is quite typical of many plasmoid encounters observed by ISEE 3 (e.g. Hones et al., 1984; Slavin et al., 1993) and GEOTAIL (Machida et al., 1994). 


\subsection{The equations of particle motions}

Test particles are introduced into the simulation box described above (Fig. 3). Their motions are computed in the dHT frame,

$$
\begin{gathered}
\frac{d \boldsymbol{v}}{d t}=\frac{q}{m} \boldsymbol{v} \times \boldsymbol{B}, \\
\frac{d \boldsymbol{r}}{d t}=\boldsymbol{v}
\end{gathered}
$$

where $m$ and $q$ are the mass and the charge of the particle, respectively. In the actual computations all the variables are normalized by the following quantities: the magnetic field by $B_{0}$ (the uniform lobe field strength at $|z| \rightarrow \infty)$, the length $r=(x, y, z)$ by the arbitrary scale $\left(L_{x}, L_{y}, L_{z}\right)$, the velocity by the Alfvén velocity $v_{A}$ (with $B=B_{0}$ ), and the time by the cyclotron time $m / q B_{0}$. Using these normalizations, the equations of motion (1) and (2) are modified into the form,

$$
\begin{aligned}
& \frac{d v}{d t}=\boldsymbol{v} \times \boldsymbol{B}, \\
& \frac{d r_{i}}{d t}=\gamma_{i} v_{i} \quad(i=x, y, z)
\end{aligned}
$$

where $\gamma$ is a dimensionless factor given by

$$
\gamma_{i}=\frac{m v_{A}}{q B_{0} L_{i}}
$$

The equations of motion are solved by a 4th-order Runge-Kutta method. For efficient computations the values of $\boldsymbol{B}$ shown in Fig. 3 are given on the $200 \times 80$ grid points in the simulation box of $10 L_{x} \times 4 L_{z}$, and their values at an arbitrary position $\boldsymbol{r}$ are derived from a spline interpolation among the nearest four grid points. In the present calculation, the kinetic energy of the particle $K_{\mathrm{HT}}=m v^{2} / 2$ in the dHT frame and the canonical momentum of the $y$ component $P_{y}=m v_{y}+q A_{y}$ should be conserved. $K_{\mathrm{HT}}$ and $P_{y}$ are watched to check the accuracy of computations every time step. After computing the motions of particles, we make a Lorentz transformation to a rest frame fixed to the earth. Since the plasmoid is moving anti-sunward with a uniform velocity $-v_{p}$ in this frame, the cross-tail electric field $E_{y}=-v_{p} B_{z}$ (normalized by $v_{A} B_{0}$ ) is emerged by this transformation. The velocity of a test particle is transformed into $\left(v_{x}-v_{p}, v_{y}, v_{z}\right)$. If not otherwise noted, we shall show hereafter particle energy $K$ in the earth rest frame. $K$ does changes in this frame due to nonzero $E_{y} \times v_{y}$.

\section{Numerical Results}

\subsection{Initial condition for the calculations}

For the test particle calculations we have to specify the dimensionless parameter $\gamma$ defined by (3). To comform the simulation results to the GEOTAIL observation (Fig. 1(a)), we assume the following unit values: $B_{0}=10 \mathrm{nT}, v_{A}=1400 \mathrm{~km} / \mathrm{s}$, hence, $E_{0}=v_{A} B_{0}=14 \mathrm{mV} / \mathrm{m}$, $m / q B_{0}=1.044 \mathrm{~s}^{-1}$ (assume protons), and $\left(L_{x}, L_{y}, L_{z}\right)=(0.917,0.917,2.294) \mathrm{R}_{\mathrm{E}}$. Thus the size of the simulation box (Fig. 3) is $9.17 \times 9.17 \mathrm{R}_{\mathrm{E}}{ }^{2}$, and this leads to the value of $\gamma_{x}, \gamma_{y}=0.25$ and $\gamma_{z}=0.1$.

We inject 4000 particles at $x=-5.0$ and $y=0.0$, and with a regular spacing interval along the $z$ axis. For the velocity distribution, we set a flowing Maxwellian shape,

$$
f(\boldsymbol{v})=\exp \left[-\frac{1}{2}\left\{\frac{\left(v_{\|}-v_{\mathrm{av}}\right)^{2}}{v_{\mathrm{th} \|^{2}}}+\frac{v_{\perp}{ }^{2}}{v_{\mathrm{th} \perp}{ }^{2}}\right\}\right]
$$


where $v_{\mathrm{av}}$ is the initial average velocity of particles, $v_{\mathrm{th}}$ is the thermal speed, subscript $\|$ and $\perp$ represent the component parallel and perpendicular to the magnetic field, respectively. We typically set $v_{\mathrm{th} \|}=0.02$ and $v_{\mathrm{th} \perp}=0.05$. In the earth's rest frame, cold ions are moving tailward with $v_{\mathrm{av}}=-0.075$ (corresponding to $-105 \mathrm{~km} / \mathrm{s}$ ). Since the velocity of the plasmoid, $v_{p}$, is set $-0.5(-700 \mathrm{~km} / \mathrm{s}), v_{\mathrm{av}}$ in the $\mathrm{dHT}$ frame is 0.425 . The gyrophase angles of the test particles are isotropically distributed.

\subsection{The association with the observation}

During the actual observation of plasmoid passages, the $z$ position of the spacecraft is fixed. In order to compare the numerical results with the observations, we have recorded various physical properties at $z=1.2$ (for 'TCR' events), 0.71 (through the peak of the plasmoid) and 0.2 (near

The association with the GEOTAIL observation

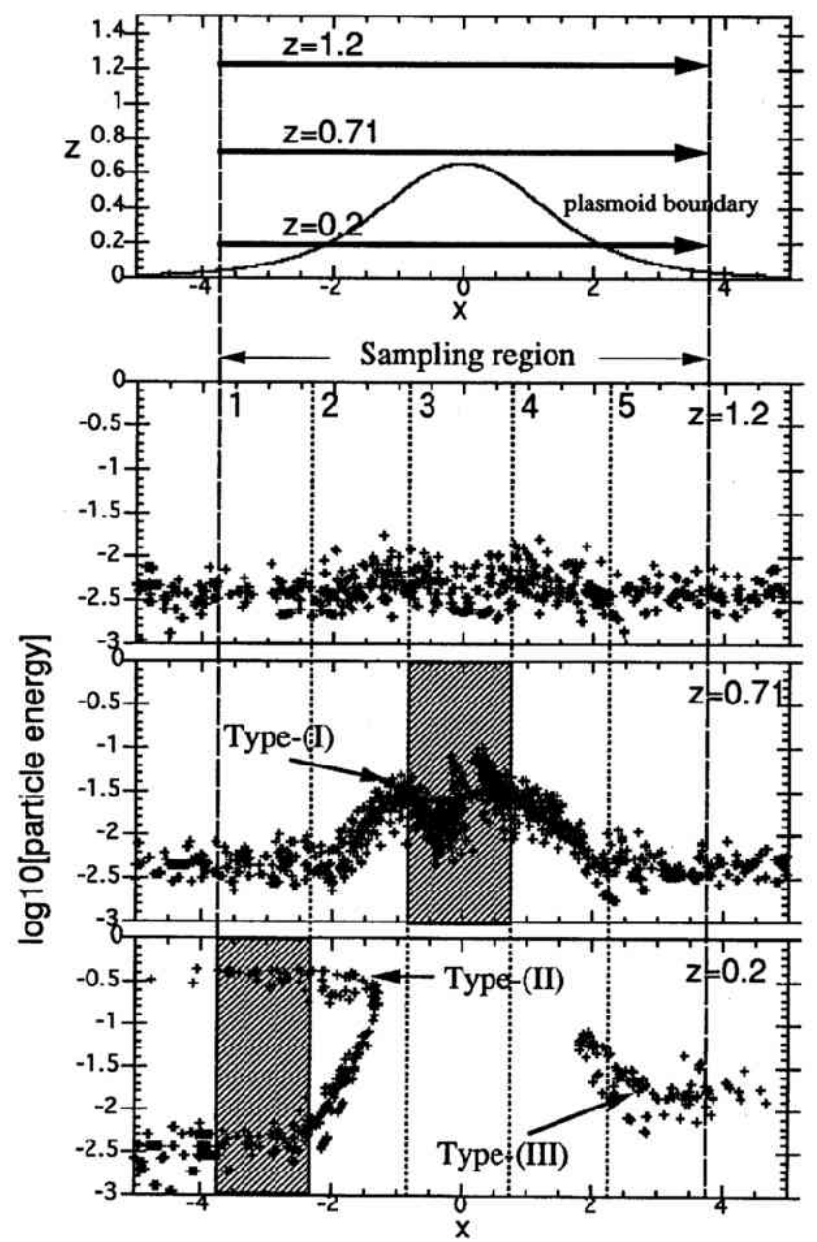

Fig. 4. Top: Projection of the plasmoid boundary onto the $x-z$ plane. Horizontal lines with arrows at $z=0.2$, 0.71 , and 1.2 show spacecraft traces through the 'sampling regions'. Bottom three panels: Variations along the $x$ axis of particle energies at the position of the spacecraft, $z=0.2,0.71$, and 1.2 . Vertical broken lines divide sampling region into five sections, location 1 to 5 . The particles in the hatched region are followed in Figs. 7 and 8 . 
the neutral sheet). The bottom three panels in Fig. 4 show the recorded particle energies as a function of $x$ for these three $z$ positions. (Note that marks in each panel come from different test particles, not showing orbits of particles.) The top panel illustrates the 'trajectory' of the spacecraft for each $z$ value (horizontal solid lines with arrows on both sides). The region between vertical dashed lines, which we call hereinafter 'sampling region', corresponds to $60 \mathrm{~s}$ duration, or $700 \mathrm{~km} / \mathrm{s} \times 60 \mathrm{~s} \sim 6.5 \mathrm{R}_{\mathrm{E}}$ during the plasmoid encounter (Figs. 1(a) and 2).

The initial particle energy $K$ was $\sim 100 \mathrm{eV}$ in each case, $z=1.2,0.71$ and 0.2 . (The particle energy $K$ is in the earth's rest frame, and normalized by $m_{p} v_{A}^{2} \sim 20 \mathrm{keV}$. We plot $\log _{10} K$ in Fig. 4.) In the sampling region at $z=1.2$ (far from the neutral sheet), the changes of particle energies are insignificant. In the other two cases $(z=0.71$ and 0.2$)$, three types of the particle energization effect have emerged. In the middle panel of the energy plot (sampled at $z=0.71$ ), the particle energies begin to increase at $x \sim-2$, reach maxima of about $\log _{10} K \sim-1$ (i.e. $\sim 2$ $\mathrm{keV}$ ) around $x \sim 0$ (the top edge of the plasmoid), and finally decrease to their original levels; we have refered to this effect as type-I energization. Type-II and type-III energization can be seen in the bottom panel (sampled at $z=0.2$ ). Type-II appears in the forward region for the particles reflected by the plasmoid and the maximum energies of particles become over $\log _{10} K \sim-0.5(>$ $6 \mathrm{keV}$ ). Type-III, which is found in $x \sim 2$, indicates that there are net energy increases after the plasmoid has moved over the spacecraft. The original energy of $\sim 100 \mathrm{eV}$ is increased to several hundreds of $\mathrm{eV}\left(\log _{10} K=-2 \sim-1.5\right)$. Note here that for the sampling region at $z=0.2$, there is no data in the middle region of the $x$ range. This is simply because particles do not have gyroradii large enough to penetrate deeply into the plasmoid.

The results shown in Fig. 4 consist of contributions from particles of different initial positions. To see the individual particle orbit, we make the following steps: First we have divided the sampling region into five sections which we will call 'locations' $1 \sim 5$ from the left-hand side of the numerical box to the right-hand side. We select the particle samples in location 1 (the initial state) and in location 3 or 5 (the most energized state). Energy spectra for these particles are

(a): for the case, $\mathrm{z}=1.2$

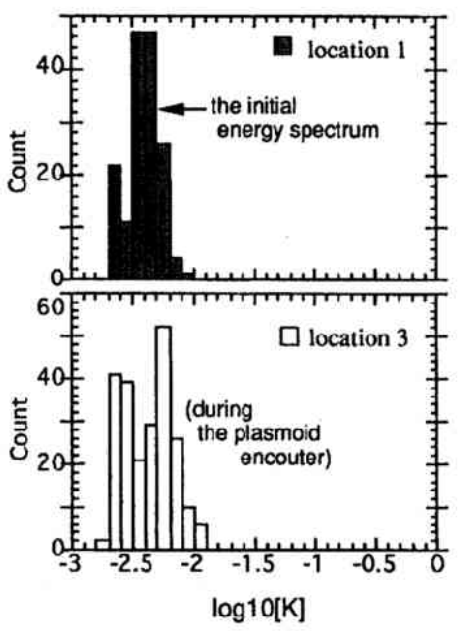

(b): for the case, $\mathrm{z}=0.71$

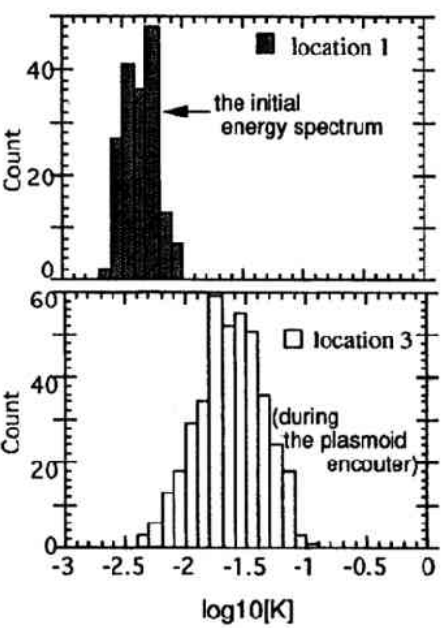

(c): for the case, $\mathrm{z}=0.2$

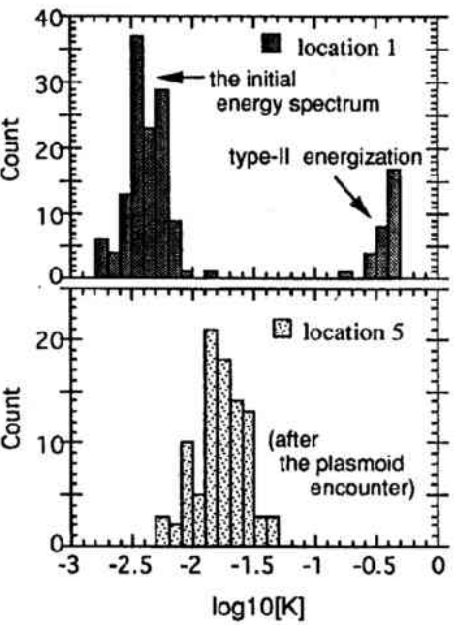

Fig. 5. Histograms of the number of particles in various position bins, 'location' 1 to 5 (obtained from Fig. 4), versus the kinetic energy in a logarithm scale (i.e. energy spectrum). The cases on the particles which are found in sampling region of Fig. 4 at (a) $z=1.2$, location 1 and 3, (b) $z=0.71$, location 1 and 3, and (c) $z=0.2$, location 1 and 5 , are displayed. 
(a): for the case, $z=1.2$

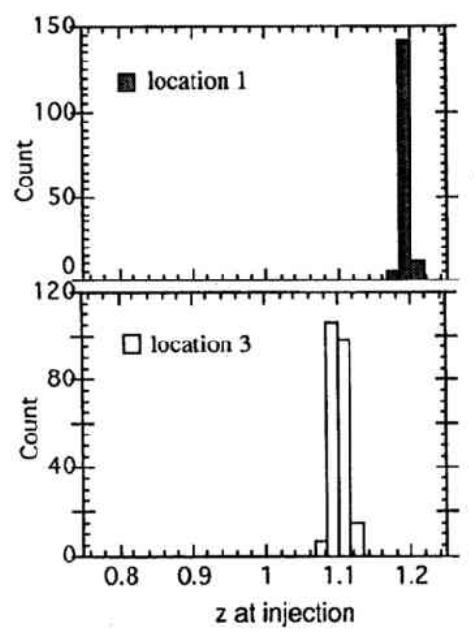

(b): for the case, $\mathrm{z}=0.71$

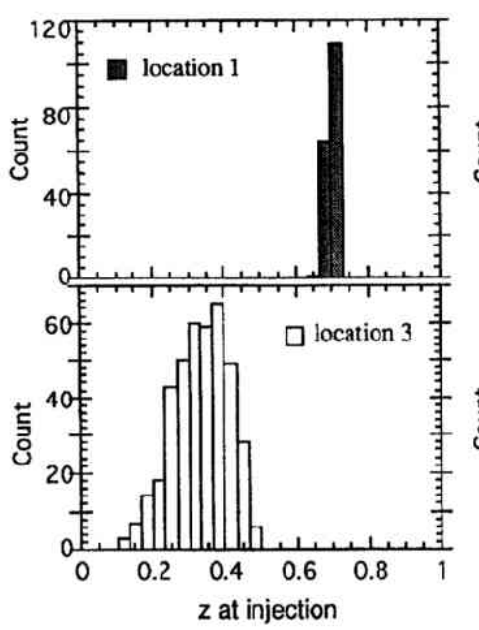

(c): for the case, $\mathrm{z}=0.2$

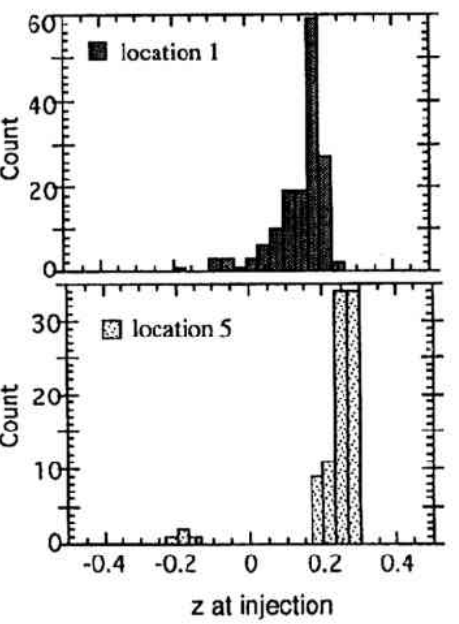

Fig. 6. Histograms for the initial injection position at the $z$ coordinate; same as the case in Fig. 5 .

shown in Fig. 5 . We then trace back the orbits of the particles sampled in Fig. 5 to their initial positions. The histograms for the initial $z$ are shown in Fig. 6.

In the upper panels of Fig. 5 , the parts of the energy spectra for $K \leq 10^{-1.5}$ show the initial energy spectra. At $z=1.2$, the energy spectrum changes little during the plasmoid passage (Fig. 5(a), lower panel). The lower panel of Fig. 6(a) shows that these particles come from $z \sim 1.1$. This means that these particles are transported by $\Delta z \sim+0.1$ during the plasmoid encounter. In Fig. 5(b) the energy spectrum of the particles in location 3 (the middle region of the plasmoid) differs from the one in location 1; particles with large energy can be found (type-I energization). Figure 6(b) shows that these energized particles are initially injected at the near neutral sheet $(z \leq 0.5)$. More complicated distrtibutions at $z=0.2$ than the other two figures are shown in Figs. 5(c) and 6(c). The energy spectrum for the particles in location 1 has two distinct peaks; the lower one corresponds to the initial energy $\sim 100 \mathrm{eV}$ and the higher one ( $\sim 6$ $\mathrm{keV}$ ) represents type-II energization. The energy spectrum in location 5 (Fig. 5(c), lower panel) indicates type-III energization; it has a larger average and a broader width than in location 1.

\subsection{Traveling of the energized particles around a plasmoid}

In order to see the particle energization processes at a plasmoid encounter, we are here concerned with trajectories of individual particles in the simulation box. For the particles moving far from the neutral sheet (e.g. the particles observed at $z=1.2$ ), a net cross-tail displacement is almost canceled out before and after the encounter with a plasmoid. These particles adiabatically trace almost along the field line (not shown here). To show the trajectories of energized particles, we select particles in the hatched regions shown in Fig. 4, i.e. sampling region of location 3 at $z=0.71$, and location 1 at $z=0.2$. We then follow their motions forward and backward in time either to the initial injection positions, or to the end of the computational run. The positions of the particles are projected onto the $x-z, x-y$, and $y-z$ plane in Figs. 7 (a) and 7 (b). In these figures, we can see that the particle motions consist of a fine scale gyration and a relatively large scale displacement (a drift motions in the $y$ direction due to the gradient and the curvature of the magnetic field).

In Figs. 7 (a) and 7(b), the orbits of many particles after passing the plasmoid are deflected 

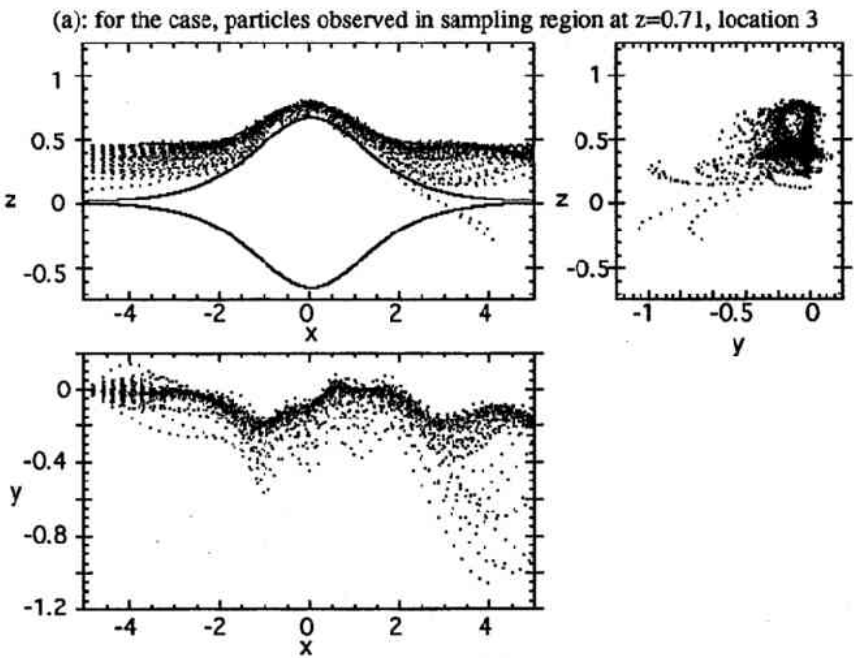

(b): for the case: at $\mathrm{z}=0.2$, location 1
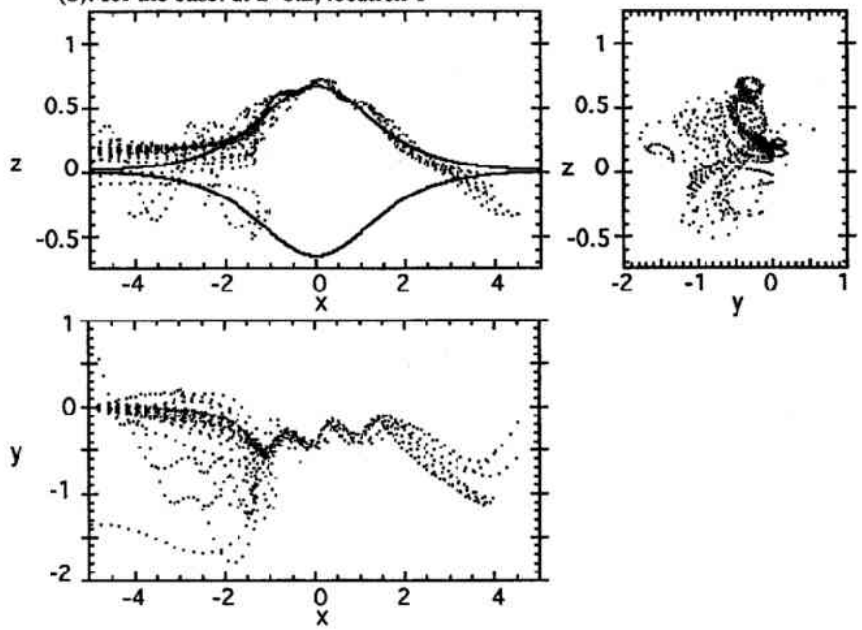

Fig. 7. The $x-z, x-y$, and $y-z$ projection of the whole orbits of the particles which are found in sampling region of Fig. 4 at (a) $z=0.71$, location 3 and (b) $z=0.2$, location 1 .

from the field lines, which have been originally traced. To get further comprehension of the particle dynamics, several physical quantities are obtained as a function of $x$ (Fig. 8). The vertical broken lines in Figs. 8(a) and 8(b) are drawn where $E_{y} \times v_{y} \sim 0$ (the fourth panel); in the areas between these neighboring lines, either $E_{y} \times v_{y}>0$ (acceleration) or $E_{y} \times v_{y}<0$ (deceleration).

As can be seen from the orbit projection onto the $x-y$ plane in Figs. 7 (a) and 7 (b), the particle orbits undergo a large cross-tail displacement in the $-y$ direction where the plasmoid is approaching. This displacement is a manifestation of the gradient and the curvature drift in the front side of the plasmoid, as noted above. A negative cross-tail electric field $E_{y}$ can accelerate these particles up to $2 \mathrm{keV}$ around the top edge of the plasmoid. What we have called the type-I energizaion in Fig. 4 is due to this acceleration process. As seen in Figs. 8(a) and 8(b), the energies are maximized at the geometrical inflection point of the field line in the leading side of the plasmoid, where the direction of the field curvature reverses, $x \sim-1.2$ (the top panels). After the corresponding reversal of the curvature drift direction, the cross-tail electric field starts to 
(a): at $\mathrm{z}=0.71$, location 3

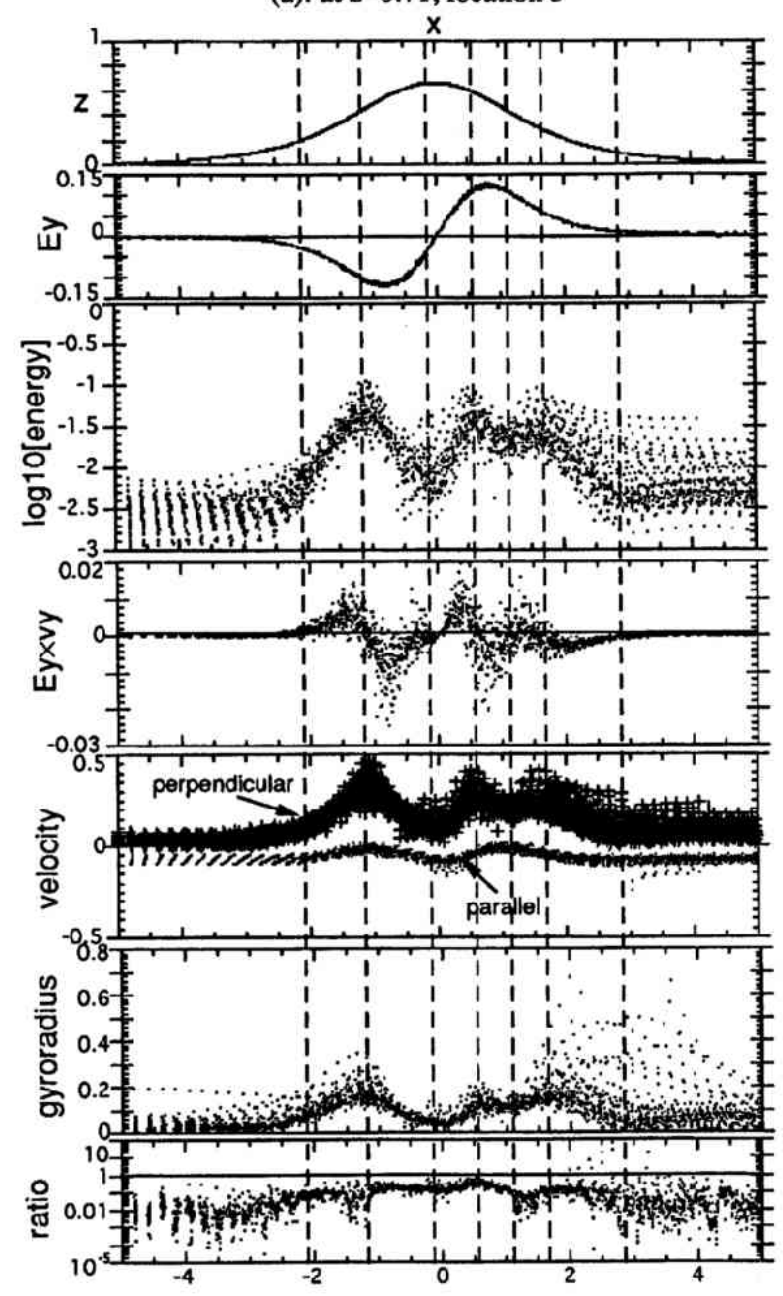

Fig. 8.

decelerate particles. Note here that the gradient drift term in particle motions can be neglected in comparison with the curvature drift term because $v_{\|}$is much larger than $v_{\perp}$ in the moving frame.

The orbit projection onto the $x-y$ plane in Fig. 7 (b) shows the reflection of some particles at the front of the plasmoid with a quite large displacement (over $1 L_{y} \sim 0.917 \mathrm{R}_{\mathrm{E}}$ ) in the cross-tail direction (type-II energization). The particles are reflected where the energies reach their maxima $(\sim 10 \mathrm{keV}$, in Fig. $8(\mathrm{~b}))$. In the velocity profiles for the reflected particles, we can see the increase of both $v_{\perp}$ and $v_{\|}$(negative value in $v_{\|}$indicates the tailward acceleration up to $\sim-1000 \mathrm{~km} / \mathrm{s}$ ).

In the trailing side of the plasmoid, particles are to be decelerated: While the particle drift motions are in the same direction as in the front side of the plasmoid, the direction of the electric field $E_{y}$ is reversed there. After exiting earthward from the plasmoid, however, the particles still keep net cross-tail displacements in the $-y$ direction, so that the effect of type-I acceleration remains (see the $x-y$ plane in Figs. $7(\mathrm{a})$ and $7(\mathrm{~b})$ ). This irreversibility is due to the non-adiabaticity in the particle behavior: At the bottom panels of Figs. 8(a) and 8(b) we show 
(b): at $\mathrm{z}=0.2$, location 1

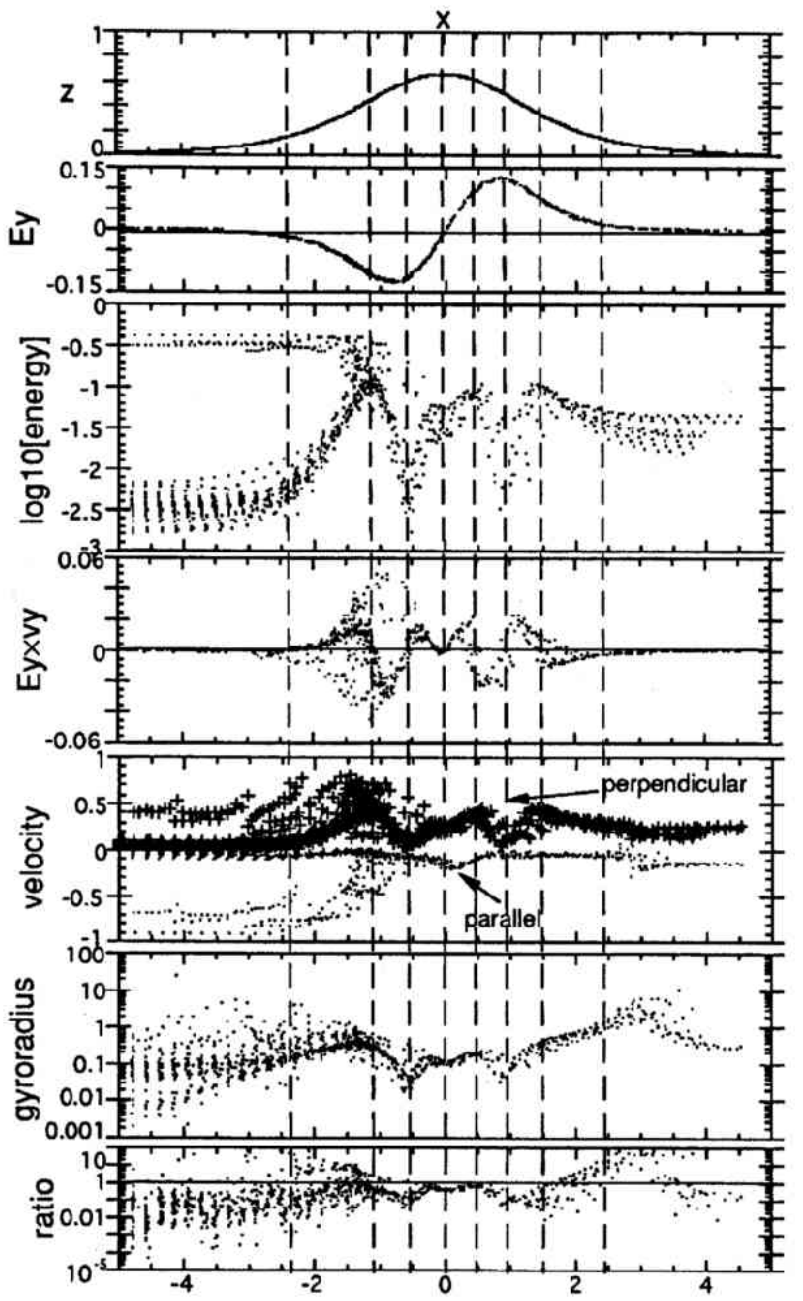

Fig. 8. Top: projection of the field line at the plasmoid boundary onto the $x-z$ plane. (Second panel to bottom) variations of the electric field at the locations of the particle shown in (a) Fig. $7 \mathrm{a}$ and (b) Fig. $7 \mathrm{~b}, E_{y}=-v_{p} B_{z}$ where $v_{p}$ is the plasmoid velocity; the particle energy with logarithmic scale, $\log K ; E_{y} \times V_{y}$ which represents the acceleration rate; velocity parallel and perpendicular to the magnetic field; normalized gyroradius, $\rho / L_{x}$; the ratio of gyroradius to the curvature radius of the field $R_{c}, \rho / R_{c}$, where $R_{c}$ is $R_{c}=1 /|(b \cdot \nabla) b| \quad(b \equiv B / B \mid)$. Vertical broken lines show the point where $E_{y} \times v_{y} \sim 0$.

the ratio, (particle gyroradius $\rho$ )/(field curvature radius $R_{c}$ ) as a measure of non-adiabaticity (Gray and Lee, 1982). Since these ratios become of $\sim 1$ after the type-I energization, the particle magnetic moments are not conserved. In other words, the particles are pitch-angle scattered and heated there (type-III energization, we call).

To see the effect of non-adiabaticity further, we define a parameter,

$$
n_{g}=\frac{a}{v_{\|}(x=0)} / \frac{2 \pi m}{q|B(x=0)|}
$$

where $a$ is defined by the extent of region between two inflection points on the plasmoid boundary. We inject a particle at $(x, y, z)=(-5.0,0.0,0.25)$ with a velocity, $\left(v_{x}, v_{y}, v_{z}\right)=(0.425,0.0,0.0)$. 
Values of $v_{\|}$(in the dHT frame) and $B$ are taken when the particle crosses over the peak of plasmoid, $x=0$. The value of $n_{g}$ roughly represents the number of the gyrations during the passage of the central region of the plasmoid. The energization rate, $R=K(x=5) / K(x=$ $-5)$ is a ratio between energies before and after the interaction with the plasmoid. Figure 9 shows the plot of $n_{g}-R$ for same topology but for various sizes of plasmoids; $\left(L_{x}, L_{y}, L_{z}\right)$ is $(0.229,0.229,0.573) \mathrm{R}_{\mathrm{E}}$ for the minimum and $(22.9,22.9,57.3) \mathrm{R}_{\mathrm{E}}$ for the maximum. The small number of $n_{g}$, i.e. for a small plasmoid, yields scattering features (non-adiabaticity). For $n_{g}>5$, the process becomes adiabatic and the acceleration and deceleration effects are canceled before and after the plasmoid passage $(R \sim 1)$. The case shown in Figs. $4-8$ is indicated by a crossing mark.

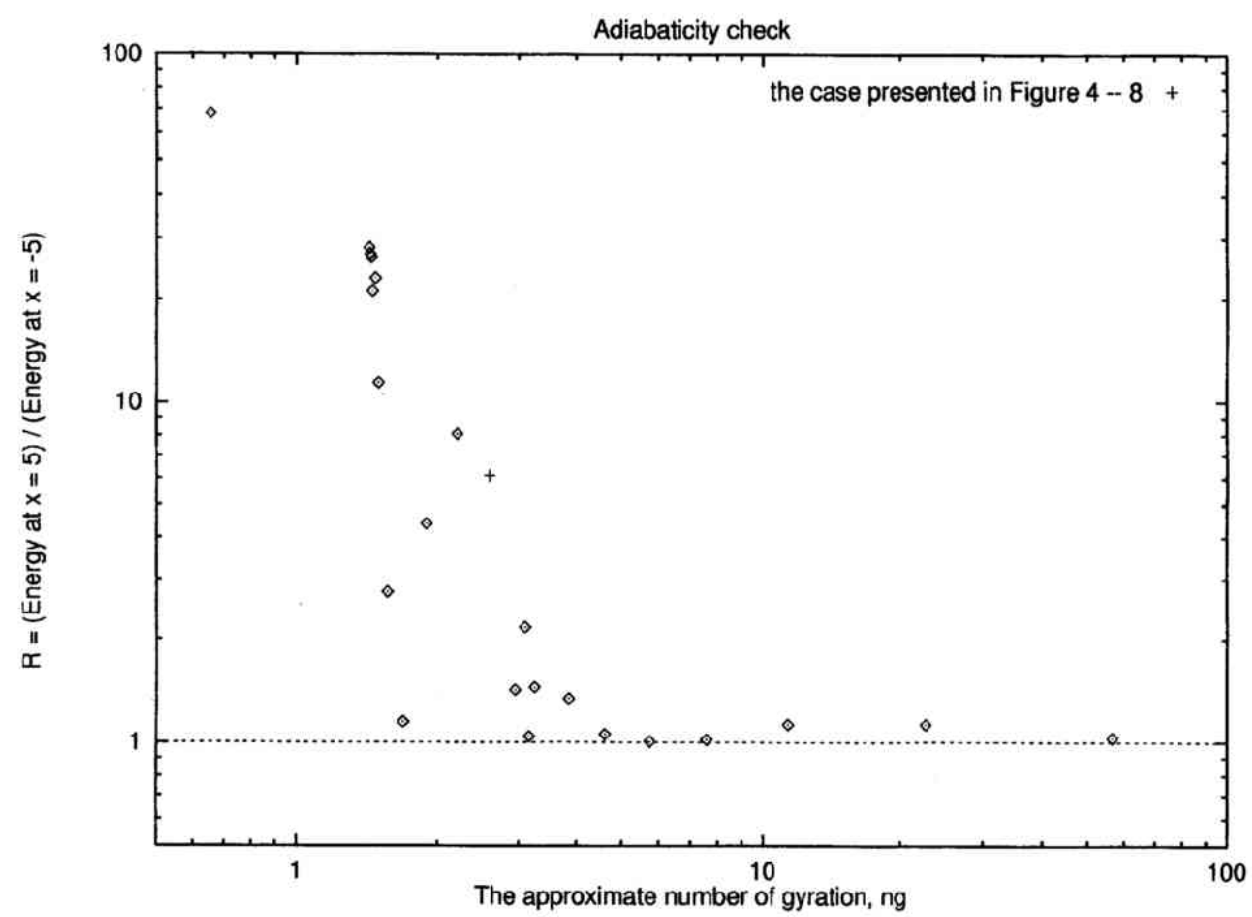

Fig. 9. The plot for the $n_{g}$ versus the energized rate, $R=K(x=5) / K(x=-5)$, which shows how the non-adiabaticity controls on the particle heating process.

\section{Summary}

In this paper we have simulated the interaction process of fast moving plasmoids with cold ion beams flowing tailward. It has been demonstrated that there are three types of the energization processes as illustrated in Fig. 10.

\subsection{Type-I energization (Fig. 10(a)):}

Cold ions, which initially flow tailward along the field lines, undergo $\boldsymbol{E} \times \boldsymbol{B}$ drifts as the plasmoid structure is approaching. At the same time, these particles also drift in the cross-tail direction due to the gradient and the curvature of the magnetic field. Since these drift motions are parallel to $\boldsymbol{E}$, these particles are accelerated. 


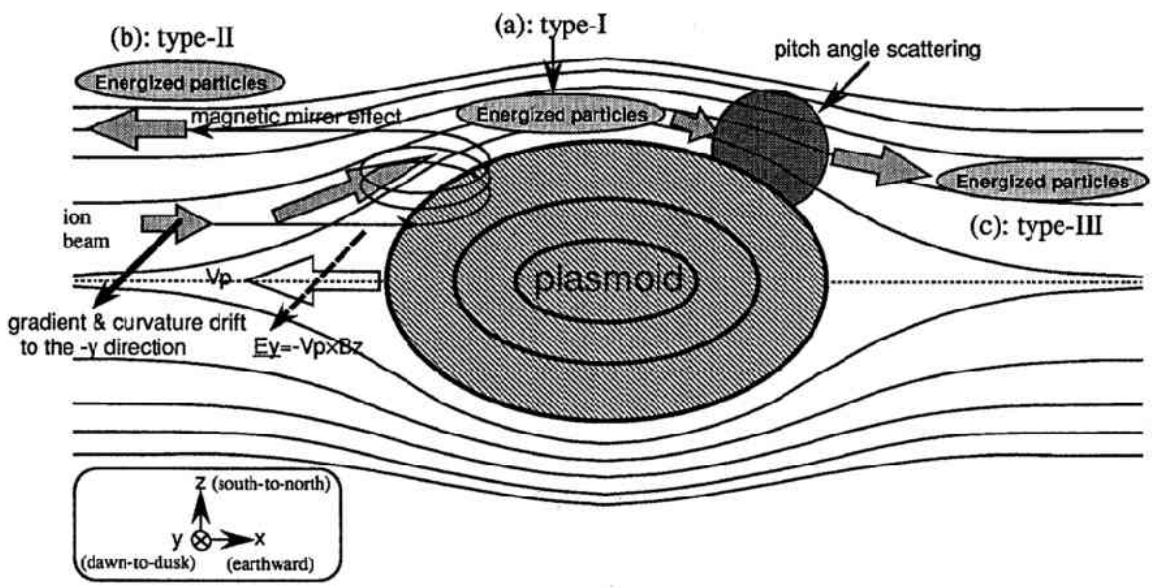

Fig. 10. Sketch for describing the process of type-I, II, and III energization. For a easy comprehension, the arrow direction for the particle motions in this figure indicates the motions in the moving frame.

\subsection{Type-II energization (Fig. 10(b)):}

As seen in Fig. 3, magnetic field lines surrounding a plasmoid are converging toward the plasmoid. (This relates to the increase of the magnetic field magnitude, observed as 'TCR' signature in the magnetotail.) Therefore, tailward flowing particles 'feel' the plasmoid as an approaching magnetic mirror. Some particles having got the large $v_{\perp}$ are reflected by the plasmoid. This 'reflection' process can be regarded as the Fermi-type acceleration process, in which particles acquire the momentum and become highly energetic (tens of $\mathrm{keV}$ order).

\subsection{Type-III energization (Fig. 10(c)):}

The ratio of particle gyroradius to the radius of the ficld curvature becomes relatively large around the top edge of the plasmoid (the bottom panels of Fig. 8). Therefore particles accelerated enough through type-I process undergo the pitch angle scattering, so that the effect of type-I energization for these scattered particles remains as the heating after the plasmoid passage.

We see that these three processes lead to the particle energization in low energy range ( several $\mathrm{keV}-10 \mathrm{keV}$ ) around a plasmoid. Type-I and type-III energization effects can be successfully compared with the recent GEOTAIL observations. As can be seen in Fig. 1(a), the type-I effect corresponds to the event between 1336 1337 UT, and the type-III heating effect is consistent with the energy broadening after 1337 UT. The type-II was not identified yet observationally, however (Mukai, private communication). While we have neglected wave-particle interaction processes in our test particle simulation, they might play some essential roles in the heating process around plasmoids. Further investigation is needed from both theoretical and observational standpoints. We pay attention here only to the mechanism which works after the separation of the plasmoid from a near-Earth neutral line. If we observe a plasmoid acceleration event in the neighborhood of the reconnection region, we may also observe particles accelerated directly in the reconnection region. It is of interest to see the relative importance of the different acceleration mechanisms.

The authors are grateful to Dr. H. Kawano for valuable discussion. The particle and the magnetic field data, used in Figs. 1 and 2, were kindly provided by the GEOTAIL LEP and MGF team. 


\section{REFERENCES}

Baker, D. N., R. C. Anderson, and R. D. Zwickl, Average plasma and magnetic field variations in the distant magnetotail associated with near-earth substorm effects, J. Geophys. Res., 92, 71-81, 1987.

Birn, J., Quasi-steady current sheet structures with field-aligned flow, J. Geophys. Res., 97, 16817-16826, 1992.

Frank, L. A., K. L. Ackerson, and D. M. Yeager, Observations of atomic oxygen $\left(\mathrm{O}^{+}\right)$in the earth's magnetotail, J. Geophys. Res., 82, 129-134, 1977.

Gray, P. C. and L. C. Lee, Particle pitch angle diffusion due to nonadiabatic effects in the plasma sheet, J. Geophys. Res., 87, 7445-7452, 1982.

Hardy, D. A., J. W. Freeman, and H. K. Hills, Double-peaked ion spectra in the lobe plasma: Evidence for massive ions?, J. Geophys. Res., 82, 5529-5540, 1977.

Hirahara, M., T. Mukai, M. Nakamura, T. Terasawa, S. Machida, Y. Saito, T. Yamamoto, S. Kokubun, and A. Nishida, Acceleration and heating of cold ion beams in the plasma sheet boundary layer observed with GEOTAIL, Geophys. Res. Lett., 1994 (submitted).

Hones, E. W., Jr., Substorm processes in the magnetotail: Comments on 'On hot tenuous plasma, fireballs, and boundary layers in the Earth's magnetotail' by L. A. Frank, K. L. Ackerson, and R. P. Lepping, J. Geophys. Res., 82, 5633-5640, 1977.

Hones, E. W., Jr., D. N. Baker, S. J. Bame, W. C. Feldman, J. T. Gosling, D. J. McComas, R. D. Zwickl, J. A. Slavin, E. J. Smith, and B. T. Tsurutani, Structure of the magnetotail at $220 \mathrm{R}_{\mathrm{E}}$ and its response to geomagnetic activity, Geophys. Res. Lett., 11, 5-7, 1984.

Machida, S., T. Mukai, Y. Saito, T. Obara, T. Yamamoto, A. Nishida, M. Hirahara, T. Terasawa, and S. Kokubun, GEOTAIL low energy particle and magnetic field observations of a plasmoid at $\mathrm{X}_{\mathrm{GSM}}=-142 \mathrm{R}_{\mathrm{E}}$, Geophys. Res. Lett., 1994 (submitted).

Mukai, T., M. Hirahara, S. Machida, Y. Saito, T. Terasawa, and A. Nishida, Geotail observation of cold ion streams in the medium distance magnetotail lobe in the course of a substorm, Geophys. Res. Lett., 21, 1023-1026, 1994.

Sato, T., H. Matsumoto, and K. Nagai, Particle acceleration in time-developing magnetic reconnection process, $J$. Geophys. Res., 87, 6089-6097, 1982.

Scholer, M., G. Gloeckler, B. Klecker, F. M. Ipavich, D. Hovestadt, and E. J. Smith, Fast moving plasma structures in the distant magnetotail, J. Geophys. Res., 89, 6717-6727, 1984.

Scholer, M. and F. Jamitzky, Particle orbits during the development of plasmoids, J. Geophys. Res., 92, 1218112186, 1987.

Sharp, R. D., D. L. Carr, W. K. Peterson, and E. G. Shelley, Ion streams in the magnetotail, J. Geophys. Res., 86, 4639-4648, 1981.

Shelley, E. G., R. D. Sharp, and R. G. Johnson, Satellite observations of an ionospheric acceleration mechanism, Geophys. Res. Lett., 3, 654-656, 1976.

Slavin, J. A., E. J. Smith, B. T. Tsurutani, D. G. Sibeck, H. J. Singer, D. N. Baker, J. T. Gosling, E. W. Hones, Jr., and F. L. Scarf, Substorm associated traveling compression regions in the distant tail: ISEE-3 geotail observations, Geophys. Res. Lett., 11, 657-660, 1984.

Slavin, J. A., M. F. Smith, E. L. Mazur, D. N. Baker, E. W. Hones, Jr., T. Iyemori, and E. W. Greenstadt, ISEE 3 observations of traveling compression regions in the earth's magnetotail, J. Geophys. Res., 98, 15425-15446, 1993.

Sonnerup, B. U. Ö., Adiabatic particle orbits in a magnetic null sheet, J. Geophys. Res., 76, 8211-8222, 1971.

Speiser, T. W., Particle trajectories in model current sheets, 1, Analytical solutions, J. Geophys. Res., 70, 42194226, 1965.

Wagner, J. S., J. R. Kan, and S. -I. Akasofu, Particle dynamics in the plasma sheet, J. Geophys. Res., 84, 891-897, 1979. 